\title{
Different genomic changes underlie adaptive evolution in populations of contrasting history
}

S.G. Seabra, ${ }^{* 1}$ I. Fragata, ${ }^{1,2}$ M.A. Antunes, ${ }^{1}$ G.S. Faria, ${ }^{1,3}$ M.A. Santos,${ }^{1,4}$ V.C. Sousa, ${ }^{1}$ P. Simões,,${ }^{1}$ and M. Matos $^{1}$

1. cE3c - Centre for Ecology, Evolution and Environmental Changes, Faculdade de Ciências, Universidade de Lisboa, Lisboa, Portugal

2. Current address: Instituto Gulbenkian de Ciência, Oeiras, Portugal

3. Current address: School of Biology, University of St Andrews, St Andrews, UK

4. Current address: CEDOC, Faculdade de Ciências Médicas, Universidade Nova de Lisboa, Lisboa, Portugal.

*Corresponding author:

Sofia G. Seabra

sgseabra@fc.ul.pt

This is a pre-copyedited, author-produced version of an article accepted for publication in Molecular Biology and Evolution following peer review. The version of record "Sofia G Seabra, Inês Fragata, Marta A Antunes, Gonçalo S Faria, Marta A Santos, Vitor C Sousa, Pedro Simões, Margarida Matos, Different Genomic Changes Underlie Adaptive Evolution in Populations of Contrasting History, Molecular Biology and Evolution, Volume 35, Issue 3, March 2018, Pages 549-563", is available online at https://doi.org/10.1093/molbev/msx247 


\begin{abstract}
Experimental evolution is a powerful tool to understand the adaptive potential of populations under environmental change. Here, we study the importance of the historical genetic background in the outcome of evolution at the genome-wide level. Using the natural clinal variation of Drosophila subobscura, we sampled populations from two contrasting latitudes (Adraga, Portugal and Groningen, Netherlands) and introduced them in a new common environment in the laboratory. We characterized the genome-wide temporal changes underlying the evolutionary dynamics of these populations, which had previously shown fast convergence at the phenotypic level, but not at chromosomal inversion frequencies. We found that initially differentiated populations did not converge either at genome-wide level or at candidate SNPs with signs of selection. In contrast, populations from Portugal showed convergence to the control population that derived from the same geographical origin and had been long-established in the laboratory. Candidate SNPs showed a variety of different allele frequency change patterns across generations, indicative of an underlying polygenic basis. We did not detect strong linkage around candidate SNPs, but rather a small but long-ranging effect. In conclusion, we found that history played a major role in genomic variation and evolution, with initially differentiated populations reaching the same adaptive outcome through different genetic routes.
\end{abstract}

\title{
Introduction
}

Experimental evolution provides important insight into the evolutionary genetics of adaptation as it allows to follow the evolutionary trajectories of adaptive mutations on several replicate populations under a controlled environment (Bailey and Bataillon 2016). The joint use of experimental evolution and genome-wide nextgeneration sequencing analysis, that is "evolve and resequence" (Turner et al. 2011; Baldwin-Brown et al. 2014; Long et al. 2015; Schlötterer et al. 2015), is revolutionizing the field, allowing to gain a deeper understanding of the genetic and molecular basis of adaptation.

Experimental evolution studies that follow the adaptive process through time tend to report a rapid and large increase in fitness in the early stages of adaptation, followed by a slowing down in subsequent periods (Teotónio and Rose 2000; Gilligan and Frankham 2003; Simões et al. 2007; Dettman et al. 2012; Wiser et al. 2013; Lenski et al. 2015; Tenaillon et al. 2016; Lenski 2017). Evolve and resequence studies nowadays allow to link these observations to molecular changes at the genomic level. A common observation in outbred sexual systems evolving in the laboratory is that adaptive alleles rarely reach fixation (Burke et al. 2010; Burke and Long 2012; Long et al. 2015), as predicted by polygenic-trait models with time-varying selection intensities. These models predict a large initial shift in allele frequencies at multiple small-effect loci, that progressively decreases and eventually plateaus as the population matches the new environmentally induced phenotypic optimum (Chevin and Hospital 2008; Burke and Long 2012; Matuszewski et al. 2015). Several underlying mechanisms may be involved: negative epistasis that leads to the reduction of beneficial fitness effects as populations approach the optimum; antagonistic pleiotropy, causing similar fitness values between 
genetic variants of opposite effects in different life-history traits; and balancing selection limiting the effects of directional selection on a given trait (Burke et al. 2010; Sellis et al. 2011; Dettman et al. 2012).

The study of the genomic signature of quantitative trait adaptation may be particularly difficult, especially when multiple loci contribute to a trait, since each locus may only exhibit small to moderate allele frequency changes (AFCs). Replicated time series data from evolve and resequence studies may contribute to distinguish the signature of adaptation of quantitative traits (evolving to an intermediate optimum) from those changing as a result of selective sweeps (leading to fixation) or genetic drift (Franssen et al. 2017). It also allows to analyze the dynamics of AFCs, namely to detect the occurrence of plateaus at different loci expected in the evolution of polygenic traits (Chevin and Hospital 2008, see above). By simulating such type of data, Franssen et al. (2017) found three phases at the genomic level: an initial directional phase; followed by a stabilizing phase (plateau of allele frequencies); and a third phase of divergence between replicated trajectories, with loss or fixation of alleles without change of trait value. These phases arise due to a combination of the above-mentioned mechanisms. Importantly, such patterns differ from predictions of classic directional selection, underlying the hard selective sweep model (Messer and Petrov 2013; Hermisson and Pennings 2017). In the latter model, the frequency of a newly arising beneficial mutation will rapidly increase toward fixation, causing a reduction of neutral variation at closely linked sites. Interestingly, many evolve and resequence studies in sexual organisms did not find evidence of such hard sweeps (Burke et al. 2010; Burke and Long 2012; Long et al. 2015). For instance, an empirical study by Orozco-terWengel et al. (2012) described instead two classes of putatively adaptive alleles, those that continuously rise in frequency but do not reach fixation, and those that increase rapidly in the beginning and then plateau. Dominance, sign epistasis and antagonistic pleiotropy were suggested as possible genetic mechanisms underlying such plateaus.

Fitness landscapes (the map between genotypes or phenotypes and fitness) can be used to study the evolutionary dynamics of populations adapting to different environments (Orr 2005; de Visser and Krug 2014). Wright proposed the "Shifting Balance Theory" (SBT), in which selection, drift and migration allowed populations to cross fitness valleys and reach different adaptive peaks (Wright 1932; Orr 2005; but see Coyne et al. 2000 for controversies on the evolutionary relevance of SBT). In such rugged fitness landscapes, the adaptive pathways available (de Visser and Krug 2014) would be dependent upon epistatic interactions between genes (Orr 2005; Svensson and Calsbeek 2012). Evolve and resequence studies provide unprecedented power to infer the topography of the fitness landscape, for example, by analyzing genomic patterns of parallel evolution in real-time evolution experiments and by characterizing variation in evolutionary paths when populations have contrasting genetic backgrounds.

The repeatability of phenotypic and genomic evolution has been a subject of interest in evolve and resequence studies, with reported cases of genomic parallelism or convergence at different levels: nucleotide, gene, multigene or gene networks (reviewed in Spor et al. 2014; Long et al. 2015; Orgogozo 2015). The 
extent of parallelism is expected to increase in this order, since there are many possible genetic routes to reach the same phenotype (Dettman et al. 2012; Tenaillon et al. 2012, 2016; Barrick and Lenski 2013; Lang et al. 2013; Spor et al. 2014; Maharjan et al. 2015; Lenski 2017). This might be even more evident in quantitative traits, considering their polygenic basis (Barton and Keightley 2002; Mackay et al. 2009). However, genetic constraints, particularly in the network of functional and regulatory interactions, may restrict the pathways effectively taken by evolution (Lind et al. 2015). In natural systems with standing genetic variation, the degree of parallelism is high at the gene level (Long et al. 2015; Bailey and Bataillon 2016), and in some cases even at the amino acid level (Zhen et al. 2012; Parker et al. 2013). Often the same beneficial alleles are targeted in laboratorial replicates of outbred sexual systems from the same initial populations (Long et al. 2015). One example of such repeatable genetic outcomes is an evolve and resequence study in outcrossing populations of Saccharomyces cerevisiae, with highly parallel responses across replicate populations under laboratory domestication (Burke et al. 2014).

Present-day patterns of genetic variation are nevertheless the direct result of a populations' evolutionary history, in particular their encountered selective pressure and demographic changes. Thus, the course of evolution should depend not only on current selection pressures, but also on the selective history of each population (Hermisson and Pennings 2005). This means that different historical genetic backgrounds between populations (historical contingency; Travisano et al. 1995; Blount et al. 2008) may lead to diverse responses when they are put in a common environment (see Cohan and Hoffmann 1986). Despite its importance, the study of historical contingency at the genomic level has largely been neglected (Matos et al. 2015) with only a few exceptions. In a study of reverse evolution, Drosophila melanogaster populations that had evolved under diversifying selective regimes were analyzed after returning to ancestral conditions (Teotónio and Rose 2000; Teotónio et al. 2009). Evolution resulting from standing variation was found to be more repeatable than evolution resulting from de novo mutations, with adaptation proceeding through sorting and recombination of standing genetic variation at multiple loci (Teotónio et al. 2009). Nevertheless, the authors showed that the phenotypic convergence to ancestral levels reported previously (Teotónio and Rose 2000) was not fully seen at the genetic level. In contrast, Graves et al. (2017) reported extensive parallel (convergent) phenotypic and genomic responses to selection between D. melanogaster lines that had undergone different evolutionary histories, including long versus recent selective regimes. Evidence of historical contingency comes mostly from studies in microorganisms. Spor et al. (2014) showed phenotypic convergence in common environments of yeast strains from different genetic backgrounds. This convergence was attained both with recurrent mutations in the same pathways, genes, and nucleotides, as well as by mutations in different pathways. This is important since the effects of mutations may be constrained by the alleles that have been historically retained, for example, due to their impact in the overall genetic background through epistasis. Similar observations have been made in Escherichia coli where - after a large number of generations - the acquisition of a rare key innovation depended on the accumulation of specific mutations (Blount et al. 2008, 2012). Likewise, when studying adaptation of E.coli to the gut of mice, Barroso-Batista et al. (2014) found that populations had evolved the same adaptive phenotype by a variety of different 
independent mutations. In contrast, Plucain et al. (2016) found historical contingencies at the phenotypic but not at the genomic level in E. coli adapting to different environments. Although these studies mark an important first step toward understanding the impact of historical contingency on evolution at the genomic level, we are currently lacking empirical studies that investigate the impact of more contrasting evolutionary histories. Such analysis will explore at a wider scale the impact of historical contingencies on evolution at the genomic level and allow inferences on the ruggedness of fitness landscapes.

In this article, we study the effect of molecular historical contingencies of Drosophila subobscura populations when adapting to a new common (laboratory) environment. This species constitutes a wellstudied example of clinal latitudinal variation in body size and in chromosomal inversion frequencies along Europe as well as in North and South America (Prevosti et al. 1988; Huey et al. 2000; Gilchrist et al. 2004), that have been linked to thermal adaptive responses (Rego et al. 2010; Rezende et al. 2010; RodríguezTrelles et al. 2013). This naturally occurring clinal variation together with previous studies of real-time laboratory adaptation (see below) makes D. subobscura an ideal system for studying the effect of historical contingencies when adapting to a common environment. We sampled populations from Southern (Adraga, Portugal) and Northern (Groningen, Netherlands) Europe and let them evolve under identical laboratory conditions. We previously characterized the evolutionary trajectories of theses populations by following changes in life history traits and inversion frequencies across replicates at several time points (Fragata, Lopes-Cunha, et al. 2014; Fragata, Simões, et al. 2014; Fragata et al. 2016). In line with previous studies (Matos et al. 2000, 2002; Simões et al. 2007, 2008) these populations quickly improved in the laboratory in fecundity related traits (Fragata, Simões, et al. 2014; Fragata et al. 2016), and—despite the high initial differentiation between them-phenotypically converged for life-history, physiological and morphological traits in just 14 generations (Fragata, Simões, et al. 2014). Interestingly though, we found that even after 40 generations, frequencies of chromosomal inversions remained differentiated between populations (Fragata, Lopes-Cunha, et al. 2014).

Our long-term studies of lab adaptation, together with more recent analyses of real time lab evolution of populations of contrasting histories (both at the phenotypic and karyotypic level) makes Drosophila subobscura unique in the understanding of the multi-level evolutionary dynamics using the lab as a novel, common environment. It is thus timely to deepen our studies by analyzing the real time evolution of such populations at the genome-wide level. In particular, given the differences between the phenotypic and karyotypic response, the present study investigates how the phenotypic responses translate on a larger genome-wide scale. We aim to decipher the genomic changes that underlie the adaptive process, and how they differ across different genetic backgrounds. To address these points, we resequenced pools of individuals from each population (3-fold replicated), and studied the genome-wide changes across several time points during laboratory adaptation. Our main questions are: Do these initially differentiated populations converge at the genome-wide level? Or are there contrasting genetic changes between populations of different histories, suggestive of a rugged underlying fitness landscape? Are there common (or 
contrasting) genes involved in the adaptive changes between populations? How much do molecular evolutionary trajectories vary between populations and between genetic variants?

Considering the rapid and steady evolutionary rate of improvement in several life-history traits in our experimental populations, suggesting that these traits are highly polygenic, we expect an initial rapid frequency change of genetic variants followed by a possible plateau. Most importantly, considering the different biogeographical histories of our populations, it is possible that contrasting initial genetic architectures lead to disparate evolutionary genomic responses due to complex underlying fitness landscapes.

\section{Results}

We performed genome resequencing of the latitudinal populations of Adraga (Ad) and Groningen (Gro) at four time points since introduction in the laboratory (fig. 1). We also sequenced a long-established laboratory control population (TA, also derived from Adraga, in 2001) at the same time points. Overall, the 24 samples had on an average $51.3 \mathrm{M}$ paired-end reads, $68-75 \%$ aligning with the draft reference genome of $D$. subobscura that we assembled (supplementary table S1, Supplementary Material). Median depths of coverage ranged from 25 to $40 \mathrm{X}$ across all sites for each sample (supplementary table S1, Supplementary Material).

\section{Genome-Wide Diversity and Differentiation}

We obtained a total of 3,040,718 SNPs across all 24 samples, with many rare alleles within each sample (supplementary figure S1, Supplementary Material). Of all SNPs detected 53\% were fixed in at least one of the samples but polymorphic in the full dataset (across replicates and generations). A total of 319,873 SNPs were fixed in all Ad samples and 295,598 in all Gro samples.

The total number of SNPs in each population decreased during laboratory adaptation, indicating that several alleles got fixed across generations, as expected due to genetic drift and/or selection (fig. 2A; supplementary figure S2A, Supplementary Material).

At the beginning of the experiment (generation 1 and 6), both experimental and control populations showed similar mean nucleotide diversity ( $\pi$; ANOVA at generation $6 ; \mathrm{F}_{1,4}=2.92, \mathrm{p}=0.162$ for Ad vs Gro; $\mathrm{F}_{1,4}=1.2$, $p=0.335$ for Ad vs control; $F_{1,4}=5.62, p=0.077$ for Gro vs control; fig. 2B). Between generation 6 and 50, we observed a significant decrease in mean diversity both in $\mathrm{Ad}$ (from 0.099 to 0.091 ) and Gro (from 0.105 to 0.098; generation term in ANCOVA, $\mathrm{F}_{1,36}=41.03, \mathrm{p}<0.0001$ ), as expected from the loss of rare alleles. This decrease was not significantly different between latitudinal populations $\left(\mathrm{F}_{1,36}=0.34, \mathrm{p}=0.561\right.$ for latitudinal population*generation term) but differed as a function of specific chromosomes (latitudinal population*generation*chromosome interaction in ANCOVA, $\mathrm{F}_{8,36}=2.45, \mathrm{p}=0.031$ ). However, diversity values were not significantly different between chromosomes (ANCOVA, $F_{4,36}=0.84, p=0.506$ for chromosome term; supplementary figure S2B, Supplementary Material). Control populations also showed a 
slight decrease in diversity between generations (from 0.097 to 0.093 ), although not statistically significant $\left(\mathrm{F}_{1,4}=4.022, \mathrm{p}=0.115\right)$.

To assess if convergence occurred at the genome-wide level, we analyzed differentiation between populations across generations. We estimated pairwise mean $\mathrm{F}_{\mathrm{ST}}$ between samples based on all three million SNPs and performed a Principal Coordinates Analysis (PCoA) to visually assess the genome-wide differentiation between samples (fig. 3A; supplementary table S2A, Supplementary Material). We observed a clear separation between Ad and Gro in initial generations, which is consistent with populations having different biogeographic histories. From generation 6 to 50, differentiation increased both between Ad and Gro and among replicates, as can be seen by the increased distance among points. In more detail, mean $\mathrm{F}_{\text {ST }}$ between Ad and Gro was initially low (0.025 at generation 1), but increased from generation 6 to 50 from 0.028 to 0.042 (supplementary table S3, Supplementary Material). This is consistent with the PCoA and suggests divergence between Ad and Gro. It is clear from the PCoA that neither Ad nor Gro replicates fully converge to the control, even though there is evidence of some convergence for Ad, with all replicates moving toward the control quadrant (negative PC1 values and positive PC2; fig. 3A; supplementary table S3, Supplementary Material).

\section{SNPs under Selection}

We tested for SNPs under positive selection in samples from Adraga and Groningen separately. To identify these candidate SNPs we used a conservative approach. Specifically, we aimed at detecting temporal changes not expected by drift alone that were consistent in the three replicate populations. For each latitudinal population, firstly we obtained the most significant SNPs using the Cochran-Mantel—Haenszel (CMH) test; secondly we retained only the SNPs with the minor allele increasing in all three replicates; and thirdly we kept those SNPs that showed the larger changes than expected by drift alone. In order to simulate AFCs due to genetic drift, we estimated the effective population size $\left(\mathrm{N}_{\mathrm{e}}\right.$; supplementary table $\mathrm{S} 4$, Supplementary Material), from temporal changes in allele frequencies. $\mathrm{N}_{\mathrm{e}}$ was on an average 91.8 in Ad and 102.1 in Gro for the G6-G25 period and it was larger in the later period (generations G25-G50, 184.0 in Ad and 182.7 in Gro).

To test whether there were differences in the adaptive process through time, we analyzed data over a short (generation G6-G25) and a long (generation G6-G50) time period. In the short period, we identified 133 and 302 SNPs under selection (i.e., that passed the three tests) in Ad and Gro, respectively. For the longer time period, we detected 248 and 165 candidate SNPs in Ad and Gro, respectively. However, the number of common SNPs between periods was rather small, with only 31 common SNPs for Ad and 36 for Gro (two of these located in the same scaffold). Furthermore, no common candidate SNPs were found between the two latitudinal populations for either period, or considering all SNPs detected in the two periods, indicating a lack of convergence on the SNPs that respond to selection. This lack of common candidate SNPs between populations leads to nonsignificant overlap $(\mathrm{P}=1)$ in the "SuperExactTest" (Wang et al. 2015) in all comparisons. Although the actual SNPs were different, some scaffolds harbored candidate SNPs for both Ad 
and Gro: eight scaffolds when considering the short period, and ten scaffolds when considering the long period.

Based on the candidate SNPs detected for Ad (short and long period), we calculated mean $\mathrm{F}_{\mathrm{ST}}$ between all pairs of samples and visually inspected the differentiation between them using PCoA (fig. 3B; supplementary table S3 and supplementary figure S3, Supplementary Material). The PCoA shows increased differentiation across generations within Ad, as expected since we defined candidate SNPs as those showing significant changes through time, but for the same SNPs differentiation was much weaker within Gro. A similar pattern was found for the candidate SNPs detected for Gro (short and long period), with significantly higher temporal differentiation in Gro than in Ad (fig. 3C; supplementary table S3 and supplementary figure S3, Supplementary Material). In short, candidate SNPs defined for a given latitudinal population did not change so much in the other population, suggesting that the two latitudinal populations have different evolutionary responses at the genomic level. Nevertheless, we found that the differentiation across generations 6 and 50 of one latitudinal population estimated with the candidate SNPs of the other is still significantly higher than in a random set of noncandidate SNPs (supplementary figure S4; Supplementary Material).

Ad and Gro diverged across generations for all four SNP sets (both short and long period), with mean pairwise $\mathrm{F}_{\mathrm{ST}}$ between latitudinal populations significantly higher in generation 50 than in generation 6 (supplementary table S3, Supplementary Material). Differentiation between replicates within each latitudinal population also increased significantly across generations (supplementary table S3, Supplementary Material), reaching higher values than with genome-wide SNPs.

Consistent with convergence of Ad to the control population (also from Adraga), for the candidate SNPs defined for Ad (short and long period), differentiation between Ad and the control decreased through time, with a significantly lower $\mathrm{F}_{\mathrm{ST}}$ in generation 50 than in generation 6 (supplementary table S3, Supplementary Material). In contrast, Gro maintained the differentiation from the control throughout time for all four SNP sets (supplementary table S3, Supplementary Material).

\section{AFC Patterns}

In order to obtain a more detailed characterization of the adaptive process at the genomic level, we examined temporal changes of allele frequencies at the candidate SNPs. Given that for each latitudinal population, we detected very few candidate SNPs shared across time periods (short and long), we investigated the patterns of AFC separately for the two sets of SNPs for each population. To compare the response of SNPs in the initial and latter periods, we plotted AFC (frequency change of the targeted, initially minor allele) between generation 6 and 25 (allele frequency difference between generation 25 and generation 6-AFC G25-G6) against the AFC between generations 25 and 50 (AFC G50-G25) for each replicate and set of SNPs. We found a range of different patterns of variation: SNPs whose minor allele presented a steep increase in the first time period (generation 6 to 25) and stabilized or had minor fluctuations in the second period 
(generation 25 to 50); SNPs with a steady increase in both periods; and SNPs that showed a slight fluctuation in the first period and a marked increase in the second period. This variation fits, in general, a significant negative correlation between the changes in frequency of the minor allele between generations 6 and 25 and generations 25 and 50 in every case (fig. 4 and supplementary table S5, Supplementary Material). This means that, as expected, SNPs with bigger changes in frequency between generations 6 and 25 tended to present smaller changes in the following period and vice versa. Furthermore, in agreement with the differential response in the two populations, SNPs with high AFC in one population exhibited AFC values closer to zero in the other (fig. 4C - F and supplementary section IV, Supplementary Material). Note, however, that several candidate SNPs had already one allele fixed in the other population (supplementary table S6, Supplementary Material). The AFCs for the control were even smaller (data not shown). Surprisingly, few of the candidate SNPs reached fixation in the last generation analyzed, namely $<5 \%$ in $\mathrm{Ad}$ and $\leq 2 \%$ in Gro (supplementary table S6, Supplementary Material).

SNPs with signs of selection between generations 6 and 25 for a given latitudinal population showed an increase in frequency of the targeted allele in the first period, as expected from the definition of candidate SNPs for that period. But, surprisingly, a large number of these SNPs showed a subsequent decrease in allele frequency in the second period (negative values in y axis AFC G50-G25 in fig. 4), both in Ad (41\%, 47\%, and $44 \%$ of the SNPs for $\mathrm{Ad}_{1}, \mathrm{Ad}_{2}$, and $\mathrm{Ad}_{3}$, respectively) and Gro (42\%, 69\%, and $67 \%$ of the SNPs for $\mathrm{Gro}_{1}, \mathrm{Gro}_{2}$, and $\mathrm{Gro}_{3}$, respectively). These SNPs will thus not show a large change if we analyze only the initial and final generations (6 to 50), which may contribute to the low number of shared SNPs across the two time periods.

To understand if this decrease in allele frequencies in the second period was due to a reduction of the intensity of selection (e.g., increasing the relative importance of genetic drift, or sampling error in the estimates) or due to changes in the direction of selection, we analyzed whether there were consistent AFCs across replicates between generation 25 and 50 for the SNPs with indications of selection in the first period. Variation among replicates was quantified by the $\mathrm{CMH} p$ values, and this was plotted against the corresponding AFC (supplementary figure S5A for Ad and S5B for Gro). We found that SNPs with decreasing allele frequencies from generation 25 to 50 (negative values in the $\mathrm{x}$ axis) were, in general, not consistent across replicates [low- $\log (\mathrm{p})$ values], suggesting that these changes were probably due to sampling effects at those SNPs.

Although we detected different sets of candidate SNPs for Ad and Gro populations, suggesting different responses at the genome level, we tested if the dynamics of allelic frequencies changes were also different by comparing the AFC patterns, considering each population's own set of candidate SNPs. Interestingly, no significant differences were found between AFC patterns of Ad and Gro for SNP sets G6-G25 (fig. 4A and G; latitudinal population*G6-G25 interaction term, $\mathrm{p}=0.611$ ) or SNP sets G6-G50 (fig. 4B and H; latitudinal 
population*G6-G25 interaction term, $\mathrm{p}=0.497$ ), suggesting that, even though the SNPs are different, they respond to selection in a similar way in the two populations.

\section{Characterization of Candidate SNPs for Selection and Surrounding Regions}

To test if our data are consistent with the hypothesis that selection acting at a particular site affects surrounding regions, we examined whether patterns of AFC around candidate SNPs were associated with increased linkage disequilibrium. For that, we plotted mean AFC between generations 6 and 50 in 100-bp windows. We characterized the surrounding regions of candidate SNPs up to the scaffold size of our current reference genome assembly. This was compared with a "random" baseline of variation around randomly drawn SNPs from the same scaffolds and a "neutral" baseline obtained from random scaffolds without candidate SNPs. For Ad, considering the scaffolds with SNPs from the SNP set Ad G6-G50, there was a pronounced decay of AFC around the candidate SNPs, reaching values similar to the "random" variation after only 200 bp (fig. 5A). Interestingly, when analyzing the same SNPs in Gro, we also detected a slightly higher average AFC around the candidate SNP (although much lower than in Ad), suggesting that these SNPs also responded to some extent in Gro (fig. 5A, inset). The "random" baseline of Ad was significantly higher than the "neutral" baseline (Wilcoxon signed rank test, $\mathrm{W}=501,470, \mathrm{p}<0.0001$ ), suggesting that SNPs located in scaffolds harboring candidate SNPs show slightly higher AFC than SNPs in other scaffolds, which can be due to the effects of selection acting at linked sites. Similar results were obtained for the candidate SNP set Gro G6-G50 (fig. 5B), and for the other set of candidate SNPs G6-G25 (supplementary figure S6A and B, Supplementary Material).

To further characterize the genetic changes around selected variants, we analyzed changes in diversity (expected heterozygosity) between generations around the candidate SNPs (supplementary figure S7, Supplementary Material). For the scaffolds containing candidate SNPs for a given latitudinal population, there was an increase in diversity between generations 6 and 50 at the candidate SNP position (somewhat expected from the methodological approach), whereas at the flanking regions there was a decrease in diversity (supplementary figure S7C, Supplementary Material). Interestingly, consistent with a lower response in Gro at the candidate SNPs from Ad (and vice versa), Gro also showed an increase in diversity at the candidate position found for Ad.

Regarding the chromosome location of the candidate SNPs, we found major differences among latitudinal populations. The majority of SNPs showing significant differences between generations 6 and 50 for Ad are located in chromosome $\mathrm{E}$ and chromosome O (51\% and 31\% of the SNPs, respectively), whereas for Gro most are located in chromosome A (82\%), the sex chromosome (table 1). We also detected a generally stronger decrease in diversity between generations 6 and 50 in chromosome $\mathrm{E}$ for Ad and in chromosome A for Gro (supplementary figure S2, Supplementary Material), which is consistent with the distribution of candidate SNPs between chromosomes (table 1). 


\section{Types of Mutations and Gene Ontology}

From the 133 and 302 SNPs indicating selection between generations in the short period for Ad and Gro, respectively, we observed significant hits with proteins in 31 and 37 SNP regions, respectively. For the long period, we found 72 hits from 248 candidate SNPs for Ad, and 24 from 165 candidate SNPs for Gro. Several biological processes are involved (section V, supplementary figure S8, table S7 Supplementary Material). The low number of hits between candidate SNPs and proteins prevents further insight namely determining predominant biological processes in the different populations and how these relate with the observed adaptive response.

\section{Discussion}

\section{Convergence, Parallelism and Divergence}

In previous studies, our team showed that populations of Drosophila subobscura derived from contrasting European latitudes quickly converged at the phenotypic level, while adapting to a laboratorial environment (Fragata, Simões, et al. 2014). In contrast, there was a lack of convergence at the karyotypic level (Fragata, Lopes-Cunha, et al. 2014). The present evolve and resequence analysis sheds light on the genomic changes underlying our previous findings, showing that these populations, initially highly differentiated, do not converge either at the genome-wide level or at SNPs with signs of selection (candidate SNPs). We have several lines of evidence supporting this finding: 1) differentiation between Ad and Gro increased across generations; 2) there were no common candidate SNPs between Ad and Gro; 3) candidate SNPs of each latitudinal population were preferentially distributed in different chromosomes; and 4) AFCs across generations for candidate SNPs detected in one population were generally much larger and consistent than in the other population. In contrast, populations with similar histories (derived from the same geographical location, nine years before) showed similar responses at the phenotypic and genomic level (i.e., phenotypic and genomic convergence). These results highlight the importance of historical contingencies in the evolutionary responses at the genomic level in a sexual organism with high standing genetic variation. It also indicates that the initially differentiated populations followed different genetic routes during laboratory adaptation to reach convergent phenotypes for life-history, physiological, and morphological traits (Fragata, Simões, et al. 2014). Furthermore, it is in agreement with the lack of convergence seen in chromosomal inversions frequencies (Fragata, Lopes-Cunha, et al. 2014). This corresponds to a scenario of a rugged fitness landscape (Wright 1932; de Visser and Krug 2014) in which the two latitudinal populations are exploring different zones of the landscape and, due to historical contingencies (e.g., epistasis), end up in different adaptive peaks, albeit with similar fitness outcomes. We cannot ascertain if epistatic interactions between genes are enough to explain the contrasting genomic changes we observed, since other factors, such as lack of genetic variants may also contribute. It does however suggest that the evolutionary dynamics of our populations depart from Fisher's additive mode of evolution (Fisher 1930; de Visser and Krug 2014).

The simplest explanation for the lack of common candidate SNPs between latitudinal populations would be that candidate SNPs found in one latitudinal population are not variable in the other. Nevertheless, few 
candidate SNPs in one population were fixed in the other since the beginning of the experiment. Most likely, adaptation with multiple loci (i.e., polygenic adaptation) contributing to the response might explain the different genetic routes observed. This could specifically involve different genetic architectures between latitudinal populations, namely different segregating standing variants, epistasis, linkage patterns, or differential pleiotropy, which leads to contrasting correlations between genetic variants and fitness (Barrett and Hoekstra 2011).

Since we used large changes in minor allele frequency to identify signatures of selection, another explanation for the lack of common SNPs between populations is that such minor alleles were not detected in the other latitudinal population because they were major, had a slower response or were lost by drift. In fact, we found some evidence that at least some candidate SNPs detected in one latitudinal population may be responding to selection in the same direction in the other latitudinal population: 1) AFCs at the candidate SNPs of one latitudinal population also show a peak (albeit smaller) in the other population; and 2) temporal changes for candidate SNPs of a given latitudinal population were also higher in the other population than at neutral SNPs. Even though this could imply that we underestimated the number of SNPs responding to selection in both populations, the lack of allele frequency convergence between populations for the majority of candidate SNPs suggests that this is not a general pattern. It is also possible that the time elapsed is not sufficient for convergence to occur. Sampling subsequent generations will allow to test whether this is the case.

Few studies have analyzed real-time evolution at both the phenotypic and genome-wide level in sexual populations of contrasting history to characterize the role of different genetic backgrounds. One notable exception is a recent study by Graves et al. (2017), where the authors analyzed the genomes of 30 populations of Drosophila melanogaster that derived from one common ancestral population introduced in the laboratory in 1970. These populations were maintained under three selection regimes, each shared by ten populations, with half having evolved for longer time in that regime than the other half. In contrast with our results, they found both phenotypic and genome-wide convergence between the long-term and the short-term populations of the same regime. It is possible that the higher initial genetic differentiation between our populations, deriving recently from natural populations of contrasting biogeographical history, is responsible for the different conclusions between studies. Moreover, it is an open question whether a more detailed analysis of candidate SNPs would maintain the conclusion that populations are following similar genetic paths.

Several studies show that parallel/convergent molecular changes are more common at gene or gene network levels than at the nucleotide level (Dettman et al. 2012; Tenaillon et al. 2012; Orgogozo 2015). Here, we did not find genes with SNPs under selection in common between Adraga and Groningen). Surely we may be missing common genes under directional selection, partly due to the caveats mentioned above and the lack of a fully annotated genome. Future studies involving more detailed gene annotation may be important in this respect. 
Evolve and resequence studies in Drosophila have generally reported a large number of candidate genes, even in regimes under very specific stressors (reviewed in Schlötterer et al. 2015). This likely reflects the polygenic basis of the studied traits and/or the possible existence of false positives. It is however difficult to validate (by functional analyses) if detected genes are the direct targets of selection due to their large number. In our study, the new selective environment does not impose a specific stress, involving a constant mild temperature, moderate density, and short generation time. Thus, the steady evolutionary response observed (Fragata, Simões, et al. 2014; Simões et al. 2017) suggests a polygenic basis (Barton and Keightley 2002). In total, we found 781 SNPs and 143 genes involved in the adaptation of our populations. We used a conservative approach in the detection of variants under selection, trying to minimize false positives due to linkage disequilibrium. This may explain the moderate number of SNPs that we found, in contrast with a much higher number detected in the laboratory adaptation study on D. melanogaster of Orozco-terWengel et al. (2012).

We found considerable differentiation between replicate populations of the same latitudinal population, particularly at the final generation, with a stronger effect for candidate SNPs than for genome-wide SNPs. This is remarkable because we might predict limited effects of drift for candidate SNPs, particularly when considering the stringent conditions that we imposed to detect these SNPs. However, uniform selection alongside genetic drift may result in more fixations of alternate alleles causing greater divergence than drift alone (Cohan 1984; Cohan and Hoffmann 1986), particularly when selection acts on rare alleles and if the underlying genetic basis is polygenic (Cohan and Hoffmann 1989). This effect has recently been backed up experimentally by Griffin et al. (2017), who selected populations of D. melanogaster for desiccation resistance, and showed that the selected lines presented greater divergence at candidate SNPs than the control lines. Genetic drift may also affect the genetic architecture through dominance and epistatic interactions, such that similar selective conditions lead to the fixation of different alleles (de Brito et al. 2005). Our results show that, even when the same allele is being selected, there may be an important interplay between selection and drift, giving rise to different rates of frequency changes. If this allele stabilizes at different frequencies across replicates, this would indicate that besides historical contingencies, the interaction $\mathrm{drift} / \mathrm{selection}$ as well as epistatic and dominance effects may also generate rugged fitness landscapes.

It is noteworthy that our detection of candidate SNPs under selection was rather conservative, since we imposed several stringent conditions. In particular, to disentangle between drift and selection, we simulated stochastic changes according to the estimated small effective population sizes. Thus, only SNPs that consistently responded in the three replicates and that showed very large AFCs (larger than due to drift in a small population) were detected, implying that SNPs under selection with smaller changes would not be found. We note however that our purpose is not to depict the exact genetic basis of adaptation to the laboratory environment, but instead we focus on the comparison of the response of two populations of 
contrasting history. Since both populations were treated the same it is unlikely that our conservative approach incurred serious bias affecting our conclusions.

\section{Patterns of AFCs in Candidate SNPs during Adaptation}

Very few candidate SNPs were fixed in the last generation, similarly to what was found in several evolve and resequence studies (Burke et al. 2010; Orozco-terWengel et al. 2012; Graves et al. 2017). Since our study spans 50 generations, one possible explanation is that not enough time has passed for the alleles to reach fixation (incomplete sweep). However, Burke et al. (2010) found that D. melanogaster populations evolving under strong selection for accelerated development did not show signatures of classic sweeps (i.e., involving the fixation of new advantageous alleles) even after 600 generations. The same pattern was found by Graves et al. (2017) following the evolution of D. melanogaster lines from six selection regimes, some experiencing $>800$ generations of evolution. These studies support the conclusion that hard selective sweeps are not predominant in the genomic evolution of sexual populations and that time is not a limiting factor preventing fixation of advantageous alleles, unless adaptation was mainly due to new mutations. But this is not likely since adaptive evolution is expected to occur in sexual populations of relatively small size mainly through standing genetic variation. The absence of fixation is also predicted when the selective advantage of a given allele diminishes as the mean fitness of the population increases, leading to smaller AFCs, eventually preventing fixation (Chevin and Hospital 2008; Burke and Long 2012; Matuszewski et al. 2015). Such "diminishing return" is expected when the fitness function presents a peak under stabilizing selection, and has been particularly considered on Fisher's geometric model of adaptation. Under this model, adaptation involves a few mutations of large phenotypic effect and many of small effect, the first being more likely to occur farther from the optimum (Fisher 1930; Orr 2005; Tenaillon 2014). Nevertheless, how much such a model applies to sexual outbred populations remains unanswered (Long et al. 2015). According to a deterministic polygenic model of adaptation by Chevin and Hospital (2008), a given beneficial allele might not reach fixation when its selective advantage is inversely proportional to the distance to the new phenotypic optimum, because the optimum can be reached due to variants in other loci, before the beneficial allele fixes. Processes such as stabilizing selection, antagonistic pleiotropy, dominance, sign epistasis, and/or heterozygote advantage may also be involved in the nonfixation of genetic variants (Sellis et al. 2011; Orozco-terWengel et al. 2012). Finally, obviously stochastic processes may also play a role in maintaining variation, particularly selection-mutation and selection-drift balance under a polygenic basis of adaptation involving alleles of small effect (Barton and Keightley 2002; Hermisson and Pennings 2017). In particular, a slower selective response expected by the small effective population size of our populations may have contributed to nonfixation of the selected allele in the 50 generations of our study (Hermisson and Pennings 2017).

One predication arising from Fisher's geometric model is a decrease in evolutionary rate through time. This expectation applies to phenotypes, and may not be accompanied at the genotypic level, due to nonlinear relations between AFCs and fitness increase, such as epistasis, dominance and different effect sizes in 
general (Chevin and Hospital 2008). Such decoupling has been shown both by experimental evolution in microbes and through evolutionary modeling including epistasis and small environmental variations (Gordo and Campos 2013). In our study, we also did not find a consistent pattern of reduction in evolutionary rate across candidate SNPs but instead a wide range of patterns of AFC: 1) rapid allele frequency change followed by stabilization or slight variation; 2) gradual increase across generations; and 3) slow increase followed by a rapid increase. Orozco-terWengel et al. (2012) described the first two patterns when studying AFC during adaptation of three replicate populations of $D$. melanogaster to a novel environment, though not the latter. Interestingly, the third pattern that was found for some SNPs in our study, is in fact expected by the rise of frequency of a rare allele, increasing the genetic variance and thus accelerating evolutionary changes with time (Fisher 1930; Falconer and Mackay 1996). Concomitantly, we observe an average increment of diversity across generations in the candidate SNPs. It is an open question whether studying more generations would show a slowing down of evolutionary rate.

Independently of the details of evolutionary dynamics and outcome, a general expectation is that directional selection leads to a temporal increase in frequency of the selected allele. Contrary to this expectation, several of the SNPs showing signs of selection in the first period exhibited a later reversal of direction. As these later changes were not consistent across replicates for most SNPs, a likely explanation is that allele frequencies reached a plateau, as expected when the response to selection is polygenic (Burke and Long 2012), with the apparent decrease being due to sampling effects. However, a few SNPs showed consistent reversal across replicates. Possible causes are epistasis (e.g., sign epistasis) or linkage association changes (e.g., due to evolutionary changes of frequencies of inversions). Burke and Long (2012) called the attention to the fact that an upward bias estimating locus-specific effects (previously described by Göring et al. 2001) might lead to a later temporal plateauing. This effect might also contribute to an apparent reversal of direction of frequency change, as we saw here for some SNPs. Nevertheless, it is unlikely that such a pattern is due to erroneous detection of SNPs under selection, since candidate SNPs had to show consistent temporal departures from neutral expectations in the three replicates.

\section{Chromosomal Effects, Linkage Disequilibrium and Chromosomal Inversions}

We found an uneven distribution of the candidate SNPs across the five chromosomes that differed between latitudinal populations. These differences were not due to a different distribution of total SNPs across chromosomes between latitudinal populations. Interestingly, this enrichment for candidate SNPs in some chromosomes corresponds to observed large changes in frequency of some inversions located in those chromosomes, particularly of $\mathrm{A}_{2}$ in the $\mathrm{A}$ (sex) chromosome for Gro, and $\mathrm{E}_{1+2+9+12}$ in chromosome $\mathrm{E}$, and $\mathrm{O}_{3+4}$ in chromosome O for Ad (Fragata, Lopes-Cunha, et al. 2014). Here we could not map SNPs to the inversions, but an improved genome assembly will allow finding the genetic variants associated with specific chromosomal inversions in the future. This will further help to understand the evolution of inversion frequencies and their impact in genome-wide patterns. It would also be important to analyze if the genetic 
content of inversions contributes to adaptation, as suggested in a study on microsatellites in D. subobscura (Santos et al. 2016).

Lower diversity in the sex chromosome is generally attributed to the higher efficacy of purifying selection in eliminating recessive lethals. In accordance with this, Orozco-terWengel et al. (2012) found that selected alleles in D. melanogaster were substantially underrepresented on the sex chromosome. Here we found the opposite in Groningen - the A chromosome had the highest number of candidate SNPs. Groningen also showed a much higher diversity in the A chromosome than Adraga. This may be explained by the high frequency of the chromosomal arrangement $\mathrm{A}_{\mathrm{ST}}$ in Groningen that in general harbors high genetic diversity (Simões et al. 2012). Our data show that the genomic diversity patterns may be population specific as a consequence of the impact of inversions on population genetic structure and the expected linkage associated with them.

Linkage disequilibrium (LD) between alleles affects the dynamics of evolution and may explain some of the patterns observed in our study, either by constraining changes in allele frequency of beneficial mutations linked with more deleterious mutations or by the hitchhiking of neutral or small effect deleterious mutations with strong beneficial mutations resulting in false positives (Messer and Petrov 2013; Tobler et al. 2014). Given our stringent criteria, we obtained a restricted number of candidate SNPs with very strong indications of selection, limiting the number of false positives. The significance threshold in genome scans is difficult to define since we do not know the proportion of the genome under positive selection and the large number of false positives due to linkage disequilibrium is a major concern (Barrett and Hoekstra 2011; Tobler et al. 2014). In our study, we had several restrictions in analyzing possible effects of LD (pooled individuals and draft fragmented reference genome), so in order to assess linkage patterns, we characterized allele frequency and diversity changes in SNPs downstream and upstream of the candidate SNPs. We observed an increase in mean diversity between generations at candidate SNPs, expected as a consequence of an increase in an initially low frequency allele to intermediate values (Hartl and Clark 1997). In neighboring regions, observed diversity changes were not high, as expected under the likely scenario of adaptation from standing genetic variation, involving soft sweeps (Hermisson and Pennings 2005, 2017; Messer and Petrov 2013). In addition, in agreement with this scenario, we did not detect strong linkage, with the AFC dropping sharply after a few hundred bases. Similarly, evolve and resequence studies with D. melanogaster (Orozco-terWengel et al. 2012; Tobler et al. 2014; Franssen et al. 2015) found low LD around the selective site. However, over large distances, Tobler et al. (2014) and Franssen et al. (2015) found long-range hitchhiking effects, with false positives linked to selected sites, which might be related with large segregating inversions (Tobler et al. 2014). In our study, we detected a long-lasting but weak signal along the $100,000 \mathrm{bp}$ considered. This is interesting, considering that this species has a rich inversion polymorphism and most of it is maintained during adaptation to the laboratory (Fragata; Lopes-Cunha, et al. 2014; Santos et al. 2016; Simões et al. 2017). However, Franssen et al. (2015) did not observe a stronger increase of LD in genomic regions spanned by inversions. Studies involving longer scaffolds, particularly with haplotype and karyotype 
knowledge will be needed to understand longer distance linkage patterns and the possible role of inversions. Such studies will enhance our understanding of how much variation in inversions contributes to the lack of genomic convergence.

\section{Concluding Remarks}

There is a surprising lack of real-time evolution studies testing a fundamental issue in evolutionary biology: what is the impact of contrasting histories in genomic outcomes during adaptation to new environments? In this study, we fill this gap by analyzing the real-time evolution at the genome-wide level of populations of Drosophila subobscura with contrasting biogeographical history, during laboratory adaptation. We showed that populations followed different and unpredictable genetic routes to reach predictable, and similar adaptive phenotypic outcomes. Thus, while adaptation to new environments seems to be attainable from different phenotypic starting points, contrasting genetic architectures constrain how populations explore the genotypic landscape. We also found in both populations a range of trajectories that could go undetected if only initial and final stages were analyzed. In summary, our study shows that the predictability of evolution may vary across biological levels. Future studies will help elucidate the precise genetic paths involved and how and why they differed between populations.

\section{Materials and Methods}

\section{Founding and Maintenance of Laboratory Populations}

Drosophila subobscura samples were collected in late August 2010 from two low-altitude sites from contrasting latitudes of the European cline: Adraga (Portugal) and Groningen (Netherlands), from which two populations, "Ad" and "Gro", were derived in the laboratory (see Fragata, Simões, et al. 2014). Wild females (234 for Ad and 160 for Gro) were kept in separate vials as well as their offspring in the following two generations to equalize family contributions, avoiding inbreeding (see details in Fragata, Simões, et al. 2014). In the third generation an equal number of offspring of each female was randomly mixed, giving rise to the outbred populations. In the fourth generation, three replicate populations were derived (e.g., $\operatorname{Ad}_{1}, \mathrm{Ad}_{2}$, and $\mathrm{Ad}_{3}$ from the Ad population). We will use the term "latitudinal population" to refer to the three replicate populations derived from the same set of wild founder females (i.e., $\mathrm{Ad}$ to $\mathrm{Ad}_{1-3}$ and $\mathrm{Gro}$ to $\mathrm{Gro}_{1-3}$ ). Three long-established populations founded from a collection in Adraga in 2001 were used as controls (TAformerly "TW" populations-Simões et al. 2008) and maintained synchronously with the experimental populations. These populations were in the $115^{\text {th }}$ generation at the time of the founding of the new populations.

All populations were maintained under the same conditions with synchronous discrete generations of 28 days, census sizes between 500 and 1200 individuals, $12 \mathrm{~L}: 12 \mathrm{D}$ at $18^{\circ} \mathrm{C}$. Flies were kept in vials with controlled density of eggs ( $\sim 70$ eggs per vial) and adults (50 adults per vial). For each replicate population flies emerging in the first 4-5 days from a total of 24 vials were randomized using $\mathrm{CO}_{2}$ anesthesia. Eggs were 
collected when flies were 7-10 days old (around the age of peak fecundity) to found the following generation (see also Simões et al. 2007, 2008; Santos et al. 2012).

\section{DNA Extraction and Sequencing}

Flies were preserved in absolute ethanol at $-20{ }^{\circ} \mathrm{C}$, after egg collection for the next generation. We used pools of 50 individual females from each replicate population/generation: $\mathrm{Ad}_{1-3}, \mathrm{Gro}_{1-3}$. The exception was the TA population (control), where pools of the three replicate populations were done. Four generations were analyzed: $1,6,25$, and 50 (as well as the corresponding synchronous generation for TA, e.g. generation 116 corresponds to generation 1 of Ad and Gro). Before DNA extraction, the abdomen of each female was discarded to avoid contamination with egg DNA. DNA extraction was done separately on pools of ten females, using DNeasy Blood \& Tissue Kit (Qiagen) following the manufacturer's protocol but replacing buffer ATL with buffer AL. After DNA quantification, performed with Qubit 2.0 Fluorometer (Invitrogen), equal DNA quantities of each pool of 10 females were used to form the total pool of 50 females, ensuring equal representation of each individual fly.

Whole-genome paired-end sequencing of the 24 samples was carried out in four flow cell lanes of Illumina HiSeq2500 sequencing system (PE 101 cycles), aiming at an average coverage of 50X of each sample. For the initial generation (generation 1, not yet replicated), an additional pool of 94 and 75 individuals of Ad and Gro, respectively, was sequenced (supplementary section I, Supplementary Material). For these two samples, we used the corresponding to one-third of one flow cell lane of HiSeq2500.

The draft reference genome of Drosophila subobscura was assembled using a homokaryotypic line of this species (chcu) (supplementary section II, Supplementary Material).

\section{Mapping, SNP Calling and Filtering}

Quality filtering was done with trim-fastq.pl script of the PoPoolation package (Kofler, Orozco-TerWengel, et al. 2011). The paired-end reads were mapped to the reference genome using $b w a$, with the following parameters: maximum number of gap extensions (-e): 12; disallow long deletion within INT bp toward 3' end (-d): 12; maximum fraction of mismatches in each read (-n): 0.01 . The alignments were filtered to remove duplicates, using MarkDuplicates.jar from PICARD tools and to remove low quality alignments and reads not mapped, using samtools view. The mapped reads of each sample were joined in a pileup using samtools mpileup. This mpileup file was then filtered to remove indels and the surrounding bases using identify-genomic-indel-regions.pl and filter-pileup-by-gtf.pl from PoPoolation package. The mpileup file was then converted to the sync format using mpileup2sync.jar from PoPoolation2 (Kofler, Pandey, et al. 2011).

For the definition of SNPs in the 24 samples, we considered sites that: 1) were not missing in any sample; 2) had only two alleles; 3 ) had coverage between 20X and 150X; 4) had minor allele read count across all samples of at least 4; and 5) were polymorphic in at least three samples. We were not conservative in the 
allowed minimum minor allele frequency since we did not want to discard initially very low allele frequencies. These are the ones most relevant in the subsequent detection of selection, where we accounted for the error associated with allele frequency estimates by finding consistent patterns across replicates in the several steps of the analysis (see below).

\section{Diversity and Differentiation Estimates}

Since genetic diversity estimates are highly influenced by the amount of coverage, a subsampling was done to standardize the coverage at 20X using subsample-pileup.pl of PoPoolation. Diversity (expected heterozygosity, $\pi$ ) was calculated for each SNP site in the dataset, using $1-\mathrm{fA}^{2}-\mathrm{fT}^{2}-\mathrm{fC}^{2}-\mathrm{fG}^{2}$, where $\mathrm{fx}$ indicates the frequency of nucleotide $\mathrm{x}$. Differences between latitudinal populations in mean diversity across SNPs (using as raw data for each SNP in Ad and Gro the average diversity between the three replicate populations) at an early generation in the lab (generation 6) were tested by applying ANOVAs between pairs of populations (i.e., Ad vs. Gro; Ad vs. control; Gro vs. control) defining population (Ad, Gro, and control) and chromosome (A, E, J, O, U) as categorical variables.

Temporal changes in diversity (between generation 6 and 50) of the Ad and Gro latitudinal populations were analyzed by applying an ANCOVA on mean diversity values, using as categorical variables: latitudinal population (Ad, Gro), replicate $(1,2,3)$ as a random factor nested within latitudinal population, chromosome; and generation ( 6 and 50 ) as covariate, and the respective interaction terms. Temporal changes in the control population were also assessed in the same period through an ANCOVA with chromosome as categorical variable and generation as covariate. An arcsine transformation was applied to all diversity values in order to meet ANOVA assumptions. These analyses were performed using STATISTICA 13 (Dell 2015).

To estimate the differentiation between pairs of samples, we calculated $\mathrm{F}_{\mathrm{ST}}$ using $f$ st-sliding.pl from PoPoolation2 with a minimum read count of the minor allele of four across all samples. Mean $\mathrm{F}_{\mathrm{ST}}$ values across SNPs for each pair of samples were used in a Principal Coordinate Analysis (pcoa in R package ape version 3.5; http://ape-package.ird.fr/; last accessed: July 2016) to explore the differences between the samples. Differences between mean $\mathrm{F}_{\mathrm{ST}}$ values across SNPs, either among generations or among latitudinal populations, were tested with Wilcoxon signed rank test in $\mathrm{R}$.

\section{Detection of Candidate Sites for Selection}

We used several criteria to define candidate SNPs involved in the adaptive process. First, we applied a Cochran-Mantel-Haenszel (CMH) test (Mantel and Haenszel 1959) implemented in cmh-test.pl (Popoolation2 package) to find significant changes in frequency between generation 6 and generation 25 (G6-G25; or between generation 6 and 50, G6-G50), that were consistent among the three replicates in either Ad or Gro. Second, from the 4,000 most significant SNPs in each comparison (99.5\% quantile or higher) we retained only the SNPs that showed an increase in the same minor allele frequency in all three replicates (because if a minor allele decreases, drift will be more likely involved). Finally, we tested if these changes 
could be solely due to drift, by simulating AFC under a Wright—Fisher model (9,999 simulations), assuming drift and no mutation or migration. Specifically, for each replicate, simulated changes in allele frequencies took into account the estimated effective population size in the respective generations (supplementary section III, Supplementary Material). We used the allele frequencies at generation 6 as starting point. To take into account sampling effects on the estimates, we used a binomial distribution to sample 100 haplotypes with a coverage of 30X at each generation. The $\mathrm{P}$ value for each SNP was calculated using the proportion of simulations with equal or higher values than the observed final frequency of the initially minor allele. SNPs were considered as having a significant sign of selection when $\mathrm{P}$ values of simulations were $\leq 0.05$ in all three replicates.

\section{Patterns of AFC of Candidate SNPs}

In each population, we assessed the pattern of allele frequency change (AFC) of SNPs with signal of selection across generations, by plotting AFC between generation 6 and 25 against AFC between generations 25 and 50. This was done for each candidate SNP set: Ad G6-G25 (SNPs with signs of selection between generation 6 and 25 in Ad), Gro G6-G25, Ad G6-G50, and Gro G6-G50. We also evaluated allele frequency patterns for all SNP sets in the other latitudinal populations (e.g., Gro and control for SNPs under selection in Ad). We obtained partial correlations between the AFC from generation 6 to 25 and AFC from generation 25 to 50 for each latitudinal population and for each SNP set in STATISTICA. For this, we applied, for each SNP set an ANCOVA model defining AFC G50-G25 as dependent variable, AFC G25-G6 as covariate and including replicates as random factor and the interaction replicate*AFC G25-G6. We did the same analysis but comparing AFC in Ad for SNP set Ad G6-G25 versus AFC in Gro for SNP set Gro G6-G25, and AFC in Ad for SNP set Ad G6-G50 versus AFC in Gro for SNP set Gro G6-G50 (supplementary section IV, Supplementary Material).

\section{Assessing Linkage Disequilibrium}

In order to assess patterns of linkage disequilibrium for the SNPs with signs of selection (candidate SNPs), we studied the average AFCs in the adjacent regions of those candidate SNPs. For that, we calculated the average AFC (between G6 and G50) across replicates for every SNP position present in each scaffold containing a candidate SNP and then an average AFC (considering only polymorphic sites) for each 100-bp non-overlapping sliding windows up to 50,000-bp upstream and downstream of the candidate SNP. This is an arbitrary value, chosen to encompass several SNPs but not too large since some scaffolds were small (10,000 $\mathrm{bp}$ ). We did the same analysis for randomly selected SNP sites from scaffolds where the SNPs with signs of selection were located, to obtain a baseline of "random" variation. Additionally, we obtained a "neutral" baseline of variation by randomly selecting SNPs from scaffolds without candidate SNPs. The same analysis with sliding window variation was done for SNP diversity (expected heterozygosity) downstream and upstream the candidate SNP. Average values of diversity for each 100-bp window considered only SNP positions and not the invariable sites. 


\section{Localization of Candidate SNPs}

There is a high conservation of chromosomal elements among D. subobscura, D. pseudoobscura, and $D$. melanogaster (Santos et al. 2010). We thus identified the localization of each of our scaffolds on the respective chromosome by using blastn with an e-value cutoff of 1e-10 to compare against $D$. pseudoobscura (version dpse_r3.04) and D. melanogaster (version dmel_r6.10) genomes available in Flybase. However, there is no conservation within each chromosomal element when comparing these species (Santos et al. 2010). To find significant hits with proteins, we searched the SNP regions (considering 100-bp upstream and downstream the candidate SNP) on protein database nr from NCBI (version October 2015) using blastx with an e-value cutoff of 1e-10. Gene Ontology and analysis of types of mutations are detailed in Supplementary Section V (Supplementary Material).

\section{Acknowledgements}

The Whole Genome Shotgun project corresponding to the draft genome has been deposited at DDBJ/ENA/GenBank under the accession NGKO00000000. The version described in this paper is version NGKO01000000. All other files (SNP data matrix, R scripts) are available through figshare (10.6084/m9.figshare.5220688 and 10.6084/m9.figshare.5220703). This work was supported by Portuguese National Funds through 'Fundação para a Ciência e a Tecnologia' (projects PTDC/BIA- BEC/098213/2008, PTDC/BIA-BIC/2165/2012 and cE3c Unit FCT funding UID/BIA/00329/2013, grants

SFRH/BD/60734/2009 to I.F. and SFRH/BPD/86186/2012 to P.S.). We thank Miguel Lopes-Cunha for help in the laboratory, Francisco Pina-Martins for help with computing, Josiane Santos and Ana Sofia Quina for discussions, and Mauro Santos and Anthony Long for advice on the study and comments on the manuscript. We also thank the three anonymous reviewers for their constructive suggestions.

\section{References}

Bailey SF, Bataillon T. 2016. Can the experimental evolution programme help us elucidate the genetic basis of adaptation in nature? Mol Ecol. 25:203-218.

Baldwin-Brown JG, Long AD, Thornton KR. 2014. The power to detect quantitative trait loci using resequenced, experimentally evolved populations of diploid, sexual organisms. Mol Biol Evol. 31:1040-1055.

Barrett RDH, Hoekstra HE. 2011. Molecular spandrels: tests of adaptation at the genetic level. Nat Rev Genet. 12:767-780.

Barrick JE, Lenski RE. 2013. Genome dynamics during experimental evolution. Nat Rev Genet. 14:827-839.

Barroso-Batista J, Sousa A, Lourenço M, Bergman M-L, Sobral D, Demengeot J, Xavier KB, Gordo I. 2014. The first steps of adaptation of Escherichia coli to the gut are dominated by soft sweeps. PLoS Genet. 10:e1004182.

Barton NH, Keightley PD. 2002. Understanding quantitative genetic variation. Nat Rev Genet. 3:11-21. 
Blount ZD, Borland CZ, Lenski RE. 2008. Historical contingency and the evolution of a key innovation in an experimental population of Escherichia coli. Proc Natl Acad Sci. 105:7899-7906.

Blount ZD, Barrick JE, Davidson CJ, Lenski RE. 2012. Genomic analysis of a key innovation in an experimental Escherichia coli population. Nature 489:513-518.

Burke MK, Long AD. 2012. What paths do advantageous alleles take during short-term evolutionary change? Mol Ecol. 21:4913-4916.

Burke MK, Dunham JP, Shahrestani P, Thornton KR, Rose MR, Long AD. 2010. Genome-wide analysis of a long-term evolution experiment with Drosophila. Nature 467:587-590.

Burke MK, Liti G, Long AD. 2014. Standing genetic variation drives repeatable experimental evolution in outcrossing populations of Saccharomyces cerevisiae. Mol Biol Evol. 31:3228-3239.

Chevin L-M, Hospital F. 2008. Selective sweep at a quantitative trait locus in the presence of background genetic variation. Genetics 180:1645-1660.

Cohan FM. 1984. Can uniform selection retard random genetic divergence between isolated conspecific populations? Evolution 38:495-504.

Cohan FM, Hoffmann AA. 1986. Genetic divergence under uniform selection. II. Different responses to selection for knockdown resistance to ethanol among Drosophila melanogaster populations and their replicate lines. Heredity 114:145-163.

Cohan FM and Hoffman AA. 1989. Uniform selection as a diversifying force in evolution: evidence from Drosophila. Am Nat. 134: 613-637.

Coyne JA, Barton NH, Turelli M. 2000. Is Wright's shifting balance process important in evolution?. Evolution 54: 306-317.

Dell, Inc. 2015. STATISTICA (data analysis software system), version 13. http://www.statsoft.com. Last accessed: July 2016.

Dettman JR, Rodrigue N, Melnyk AH, Wong A, Bailey SF, Kassen R. 2012. Evolutionary insight from whole-genome sequencing of experimentally evolved microbes. Mol Ecol. 21:2058-2077.

de Brito RA, Pletscher LS and Cheverud JM. 2005. The evolution of genetic architecture. I. Diversification of genetic backgrounds by genetic drift. Evolution 59:2333-2342.

de Visser JAGM, Krug J. 2014. Empirical fitness landscapes and the predictability of evolution. Nat Rev Genet. 15:480-490.

Falconer DS, Mackay TFC.1996. Introduction to Quantitative Genetics. Harlow: Longmans Green.

Fisher RA. 1930. The Genetical Theory of Natural Selection. Oxford: Clarendon Press.

Fragata I, Lopes-Cunha M, Bárbaro M, Kellen B, Lima M, Santos MA, Faria GS, Santos M, Matos M, Simões P. 2014. How much can history constrain adaptive evolution? A real-time evolutionary approach of inversion polymorphisms in Drosophila subobscura. J Evol Biol. 27:2727-2738.

Fragata I, Simões P, Lopes-Cunha M, Lima M, Kellen B, Bárbaro M, Santos J, Rose MR, Santos M, Matos

M. 2014. Laboratory selection quickly erases historical differentiation. PLoS One 9:e96227.

Fragata I, Lopes-Cunha M, Bárbaro M, Kellen B, Lima M, Faria GS, Seabra SG, Santos M, Simões P, Matos M. 2016. Keeping your options open: maintenance of thermal plasticity during adaptation to a stable 
environment. Evolution, 70:195-206.

Franssen SU, Nolte V, Tobler R, Schlötterer C. 2015. Patterns of linkage disequilibrium and long range hitchhiking in evolving experimental Drosophila melanogaster populations. Mol Biol Evol. 32:495509.

Franssen SU, Kofler R, Schlötterer C. 2017. Uncovering the genetic signature of quantitative trait evolution with replicated time series data. Heredity 118:42-51.

Gilchrist GW, Huey RB, Balanya J, Pascual M, Serra L. 2004. A time series of evolution in action: a latitudinal cline in wing size in South American Drosophila subobscura. Evolution 58:768-780.

Gilligan DM, Frankham R. 2003. Dynamics of genetic adaptation to captivity. Conserv Genet. 4:189-197.

Gordo I, Campos PRA. 2013. Evolution of clonal populations approaching a fitness peak. Biol Lett. 9:20120239.

Göring HHH, Terwilliger JD, Blangero J. 2001. Large upward bias in estimation of locus-specific effects from genomewide scans. Am J Hum Genet. 69:1357-1369.

Graves JL, Hertweck KL, Phillips MA, Han M V, Cabral LG, Barter TT, Greer LF, Burke MK, Mueller LD, Rose MR. 2017. Genomics of parallel experimental evolution in Drosophila. Mol Biol Evol. 34:831842.

Griffin PC, Hangartner SB, Fournier-Level A, Hoffmann AA. 2017. Genomic trajectories to desiccation resistance: convergence and divergence among replicate selected Drosophila lines. Genetics 205:871890.

Hartl DL, Clark AG. 1997. Principles of population genetics. Sunderland, MA, USA: Sinauer Associates.

Hermisson J, Pennings PS. 2005. Soft sweeps: molecular population genetics of adaptation from standing genetic variation. Genetics 169:2335-2352.

Hermisson J, Pennings PS. 2017. Soft sweeps and beyond: understanding the patterns and probabilities of selection footprints under rapid adaptation. Methods Ecol Evol 8: 700-716.

Huey RB, Gilchrist GW, Carlson ML, Berrigan D, Serra L. 2000. Rapid evolution of a geographic cline in size in an introduced fly. Science 287:308-309.

Kofler R, Orozco-terWengel P, De Maio N, Pandey RV, Nolte V, Futschik A, Kosiol C, Schlötterer C. 2011. PoPoolation: A toolbox for population genetic analysis of next generation sequencing data from pooled individuals. PLoS One 6:e15925.

Kofler R, Pandey RV, Schlötterer C. 2011. PoPoolation2: identifying differentiation between populations using sequencing of pooled DNA samples (Pool-Seq). Bioinformatics 27:3435-3436.

Lang GI, Rice DP, Hickman MJ, Sodergren E, Weinstock GM, Botstein D, Desai MM. 2013. Pervasive genetic hitchhiking and clonal interference in forty evolving yeast populations. Nature 500:571-574.

Lenski RE. 2017. Convergence and divergence in a long-term experiment with bacteria. Am Nat. 190:S57S68.

Lenski RE, Wiser MJ, Ribeck N, Blount ZD, Nahum JR, Morris JJ, Zaman L, Turner CB, Wade BD, Maddamsetti R, et al. 2015. Sustained fitness gains and variability in fitness trajectories in the longterm evolution experiment with Escherichia coli. Proc R Soc B Biol. Sci. 282:20152292. 
Lind PA, Farr AD, Rainey PB. 2015. Experimental evolution reveals hidden diversity in evolutionary pathways. eLife 4:1-17.

Long A, Liti G, Luptak A, Tenaillon O. 2015. Elucidating the molecular architecture of adaptation via evolve and resequence experiments. Nat Rev Genet. 16:567-582.

Mackay TF, Stone EA, Ayroles JF. 2009. The genetics of quantitative traits: challenges and prospects. Nat Rev Genet. 10:565-577.

Maharjan RP, Liu B, Feng L, Ferenci T, Wang L. 2015. Simple phenotypic sweeps hide complex genetic changes in populations. Genome Biol Evol. 7:531-544.

Mantel N, Haenszel W. 1959. Statistical aspects of the analysis of data from retrospective studies of disease. J Natl Cancer Inst. 22: 719-748.

Matos M, Rose MR, Rocha Pité MT, Rego C, Avelar T. 2000. Adaptation to the laboratory environment in Drosophila subobscura. J Evol Biol. 13:9-19.

Matos M, Avelar T, Rose MR. 2002. Variation in the rate of convergent evolution: Adaptation to a laboratory environment in Drosophila subobscura. J Evol Biol. 15:673-682.

Matos M, Simões P, Santos MA, Seabra SG, Faria GS, Vala F, Santos J, Fragata I. 2015. History, chance and selection during phenotypic and genomic experimental evolution: replaying the tape of life at different levels. Front Genet. 6:1-4.

Matuszewski S, Hermisson J, Kopp M. 2015. Catch me if you can: adaptation from standing genetic variation to a moving phenotypic optimum. Genetics 200:1255-1274.

Messer PW, Petrov DA. 2013. Population genomics of rapid adaptation by soft selective sweeps. Trends Ecol Evol. 28:659-669.

Orgogozo V. 2015. Replaying the tape of life in the twenty-first century. Interface Focus 5:20150057.

Orozco-terWengel P, Kapun M, Nolte V, Kofler R, Flatt T, Schlötterer C. 2012. Adaptation of Drosophila to a novel laboratory environment reveals temporally heterogeneous trajectories of selected alleles. Mol Ecol. 21:4931-4941.

Orr HA. 2005. The genetic theory of adaptation: a brief history. Nat Rev Genet. 6:119-127.

Parker J, Tsagkogeorga G, Cotton JA, Liu Y, Provero P, Stupka E, Rossiter SJ. 2013. Genome-wide signatures of convergent evolution in echolocating mammals. Nature 502:228-231.

Plucain J, Suau A, Cruveiller S, Médigue C, Schneider D, Le Gac M. 2016. Contrasting effects of historical contingency on phenotypic and genomic trajectories during a two-step evolution experiment with bacteria. BMC Evol Biol. 16:86.

Prevosti A, Ribo G, Serra L, Aguade M, Balaña J, Monclus M, Mestres F. 1988. Colonization of America by Drosophila subobscura: Experiment in natural populations that supports the adaptive role of chromosomal-inversion polymorphism. Proc Natl Acad Sci USA. 85:5597-5600.

Rego C, Balanyà J, Fragata I, Matos M, Rezende EL, Santos M. 2010. Clinal patterns of chromosomal inversion polymorphisms in Drosophila subobscura are partly associated with thermal preferences and heat stress resistance. Evolution 64:385-397.

Rezende E, Balanyà J, Rodríguez-Trelles F, Rego C, Fragata I, Matos M, Serra L, Santos M. 2010. Climate 
change and chromosomal inversions in Drosophila subobscura. Clim Res. 43:103-114.

Rodríguez-Trelles F, Tarrio R, Santos M. 2013. Genome-wide evolutionary response to a heat wave in Drosophila. Biol Lett. 9:20130228.

Santos J, Serra L, Solé E, Pascual M. 2010. FISH mapping of microsatellite loci from Drosophila subobscura and its comparison to related species. Chromosom Res. 18:213-226.

Santos J, Pascual M, Simões P, Fragata I, Lima M, Kellen B, Santos M, Marques A, Rose MR, Matos M. 2012. From nature to the laboratory: the impact of founder effects on adaptation. J Evol Biol. 25:26072622.

Santos J, Pascual M, Fragata I, Simões P, Santos MA, Lima M, Marques A, Lopes-Cunha M, Kellen B, Balanyà $\mathrm{J}$, et al. 2016. Tracking changes in chromosomal arrangements and their genetic content during adaptation. J Evol Biol. 29:1151-1167.

Schlötterer C, Kofler R, Versace E, Tobler R, Franssen SU. 2015. Combining experimental evolution with next-generation sequencing: a powerful tool to study adaptation from standing genetic variation. Heredity 114:431-440.

Sellis D, Callahan BJ, Petrov DA, Messer PW. 2011. Heterozygote advantage as a natural consequence of adaptation in diploids. Proc Natl Acad Sci USA. 108:20666-20671.

Simões P, Rose MR, Duarte A., Gonçalves R, Matos M. 2007. Evolutionary domestication in Drosophila subobscura. J Evol Biol. 20:758-766.

Simões P, Santos J, Fragata I, Mueller LD, Rose MR, Matos M. 2008. How repeatable is adaptive evolution? The role of geographical origin and founder effects in laboratory adaptation. Evolution 62:1817-1829.

Simões P, Calabria G, Picão-Osório J, Balanyà J, Pascual M. 2012. The genetic content of chromosomal inversions across a wide latitudinal gradient. PLoS One 7:e51625.

Simões P, Fragata I, Seabra SG, Faria GS, Santos MA, Rose MR, Santos M, Matos M. 2017. Predictable phenotypic, but not karyotypic, evolution of populations with contrasting initial history. Scientific Reports. 7:913.

Spor A, Kvitek DJ, Nidelet T, Martin J, Legrand J, Dillmann C, Bourgais A, de Vienne D, Sherlock G, Sicard D. 2014. Phenotypic and genotypic convergences are influenced by historical contingency and environment in yeast. Evolution 68:772-790.

Svensson E and Calsbeek R. 2012. The Adaptive Landscape in Evolutionary Biology. Oxford University Press, Oxford.

Tenaillon O, Rodríguez-Verdugo A, Gaut RL, McDonald P, Bennett AF, Long AD, Gaut BS. 2012. The molecular diversity of adaptive convergence. Science 335:457-461.

Tenaillon O. 2014. The Utility of Fisher's Geometric Model in Evolutionary Genetics. Annu Rev Ecol Evol Syst. 45:179-201.

Tenaillon O, Barrick JE, Ribeck N, Deatherage DE, Blanchard JL, Dasgupta A, Wu GC, Wielgoss S, Cruveiller S, Médigue C, et al. 2016. Tempo and mode of genome evolution in a 50,000-generation experiment. Nature 536:165-170.

Teotónio H, Rose MR. 2000. Variation in the reversibility of evolution. Nature 408:463-466. 
Teotónio H, Chelo IM, Bradić M, Rose MR, Long AD. 2009. Experimental evolution reveals natural selection on standing genetic variation. Nat Genet. 41:251-257.

Tobler R, Franssen SU, Kofler R, Orozco-terWengel P, Nolte V, Hermisson J, Schlötterer C. 2014. Massive habitat-specific genomic response in D. melanogaster populations during experimental evolution in hot and cold environments. Mol Biol Evol. 31:364-375.

Travisano M, Mongold JA, Bennett AF, Lenski RE. 1995. Experimental tests of the roles of adaptation, chance, and history in evolution. Science 267:87-90.

Turner TL, Stewart AD, Fields AT, Rice WR, Tarone AM. 2011. Population-based resequencing of experimentally evolved populations reveals the genetic basis of body size variation in Drosophila melanogaster. PLoS Genet. 7:e1001336.

Wang M, Zhao Y, Zhang B. 2015. Efficient Test and Visualization of Multi-Set Intersections. Scientific Reports 5: 16923.

Wiser MJ, Ribeck N, Lenski RE. 2013. Long-term dynamics of adaptation in asexual populations. Science 342:1364-1367.

Wright S. 1932. The roles of mutation, inbreeding, crossbreeding and selection in evolution. In: Proceedings of the sixth international congress of genetics, Volume I, p. 356-366.

Zhen Y, Aardema ML, Medina EM, Schumer M, Andolfatto P. 2012. Parallel molecular evolution in an herbivore community. Science 337:1634-1637.

\section{Figure legends}

Figure 1- Experimental design of the study. We performed genome resequencing for pools of 50 individuals from the latitudinal populations (Adraga - triangles, Groningen - squares, Control - diamonds) at four different generations (the generations numbers are marked for each latitudinal population). At generation 1 populations were not yet replicated. After generation 1 replicate populations are marked as: continuous line replicate 1; dashed line - replicate 2; dotted line - replicate 3. Control populations were sequenced synchronously, pooling all three replicate populations together at each time point analyzed.

Figure 2 - Estimates for each latitudinal population and generation of (A) number of SNPs, and (B) mean heterozygosity. Bars are mean values of replicates and error bars represent standard errors.

Figure 3 - Principal Coordinate Analysis of mean pairwise $\mathrm{F}_{\mathrm{ST}}$ estimates with the 3 million genome-wide SNP panel (A), with the 248 SNPs significant for selection in Adraga for the long period G6-G50 (B), and with the 165 SNPs significant for selection in Groningen for G6-G50 (C). Triangles: Adraga; squares: Groningen; diamonds: control populations. Increasing generations with darker shades of gray. Arrows join generations of the same replicate (continuous line - replicate 1; dashed line - replicate 2; dotted line replicate 3). PCoA for the SNP sets G6-G25 are found in Supplementary figure S3, Supplementary Material. 
Figure 4 - Allele frequency change between G6 and G25 (AFC G25-G6) plotted against AFC between G25 and G50 (AFC G50-G25) for the SNP sets Ad G6-G25 (A, E), Ad G6-G50 (B, F), Gro G6-G25 (C, G), and Gro G6-G50 (D, H) for each latitudinal population, Adraga (A - D) and Groningen (E - H). See supplementary table S5, Supplementary Material for ANCOVA tests.

Figure 5 - Mean Allele Frequency Change (AFC) between G6 and G50 of regions downstream and upstream candidate SNPs (at 0 bp) in windows of 100 bp. (A) SNP set Ad G6-G50; (B) SNP set Gro G6-G50. Black Adraga; gray - Groningen; Thick line - variation around candidate SNPs; thin line - variation around random SNPs from scaffolds with candidate SNPs ("random” baseline); Discontinuous lines - variation around random SNPs from scaffolds without candidate SNPs ("neutral” baseline).

Table 1 - Number of significant SNPs (and percentage in parenthesis) found in each chromosome for each of the comparisons.

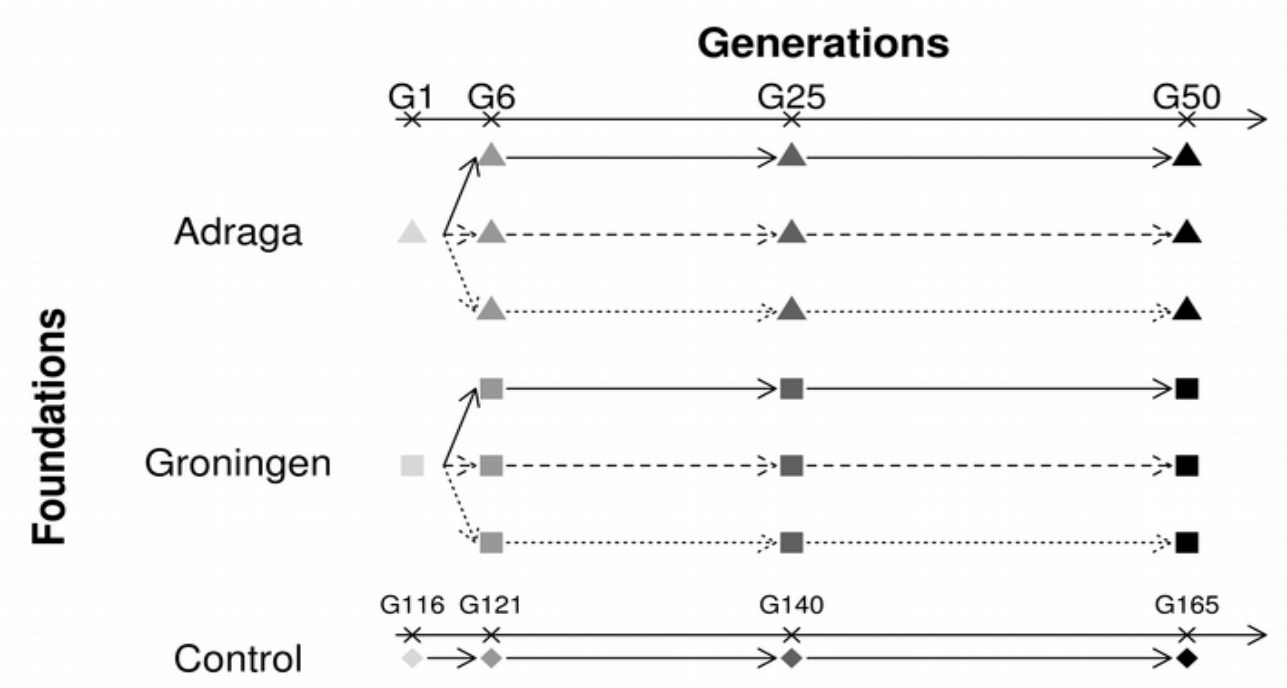

Fig. 1 

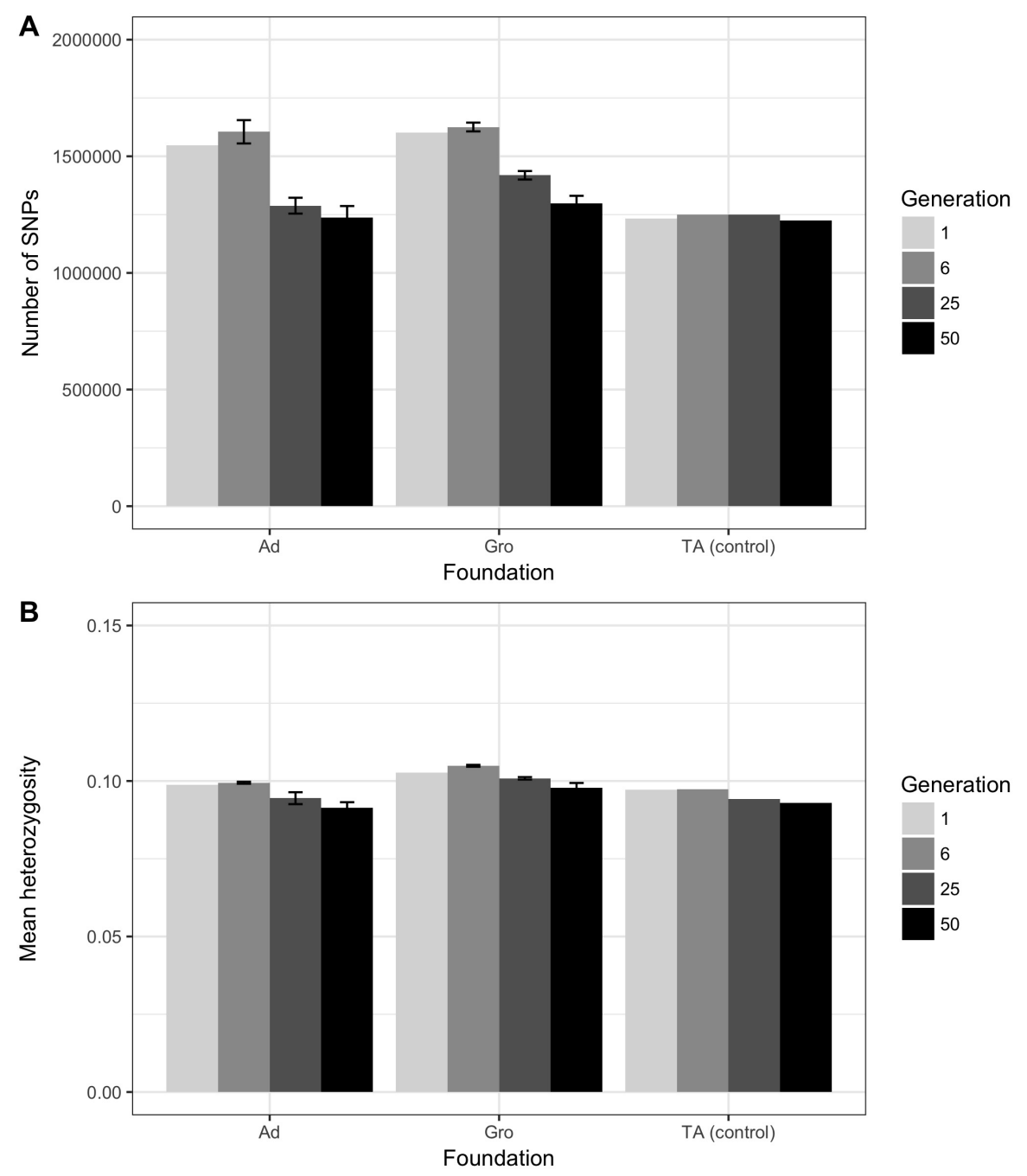

Fig. 2
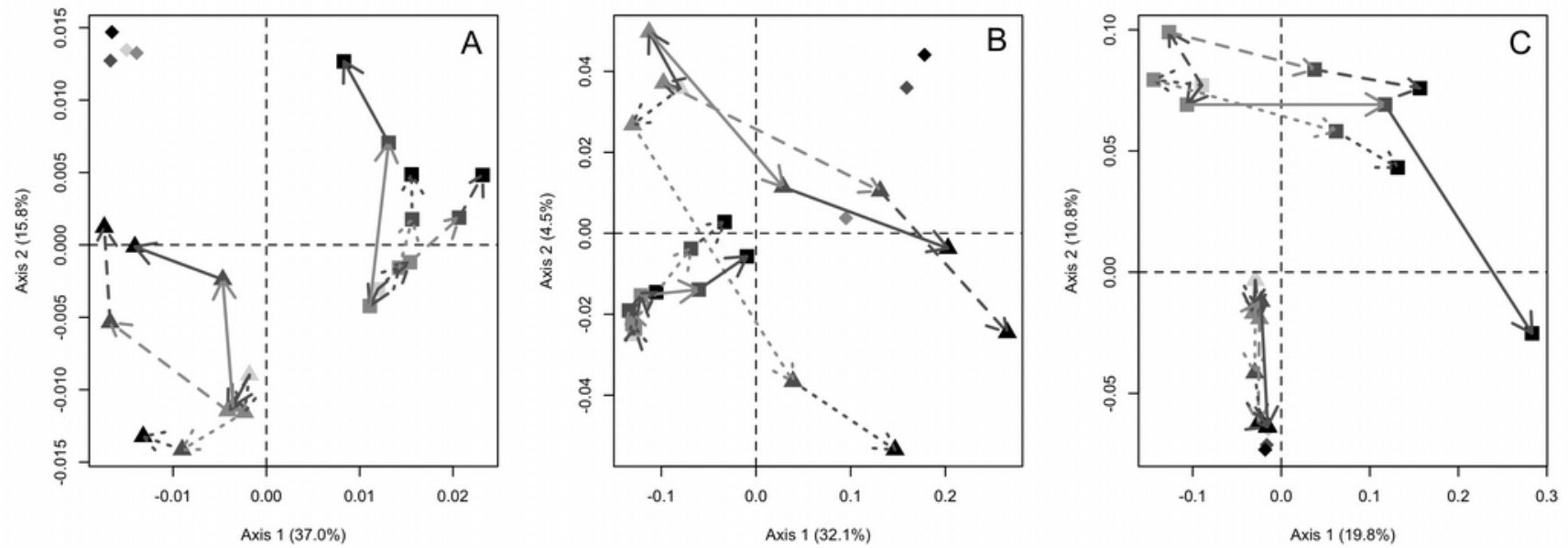

Fig. 3 


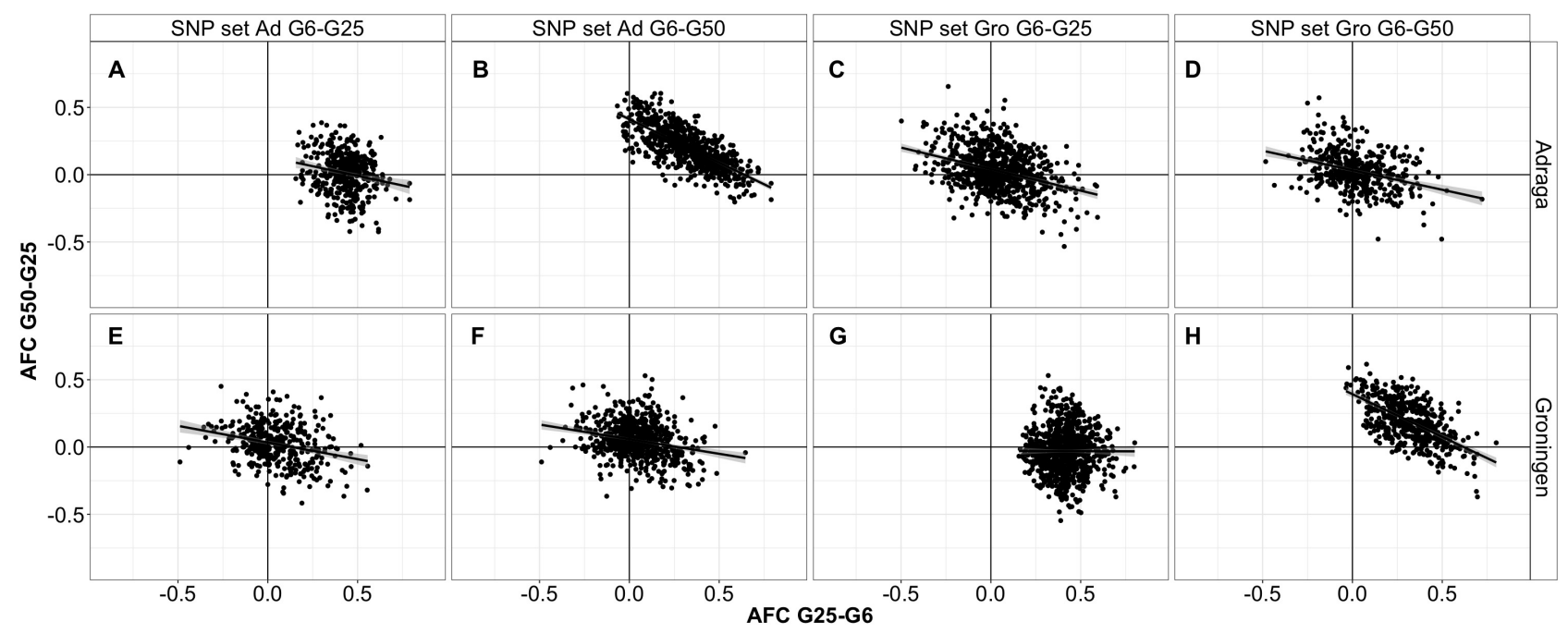

Fig. 4
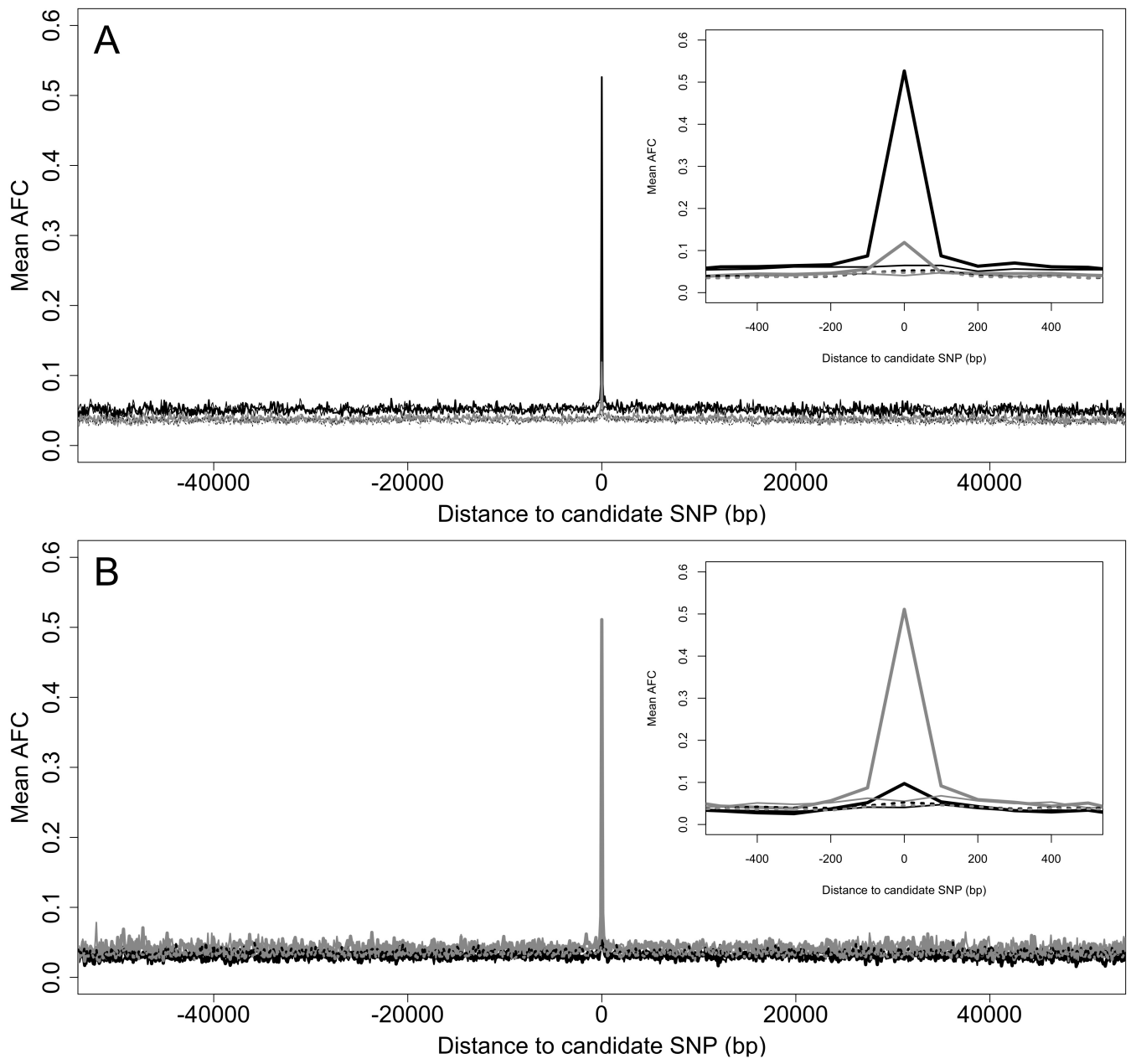

Fig. 5 